\title{
Analysis and perception: architectural pedagogy for environmental sustainability
}

\author{
Y. Luckan \\ Department of Architecture, \\ Durban University of Technology, South Africa
}

\begin{abstract}
Theories and concepts of environmental sustainability in architectural education weigh heavily toward the analysis of scientific data and conversely largely ignores the value of perception as an analytical tool. Architects, urban designers and students have been largely influenced by these indicators and much attention and time is spent on energy calculations and performance analysis. The ability to understand architecture and place as responsive to people, place and time has been undermined, hence an antithesis to sustainable architecture emerges. The analysis of place is often based on abstract perceptual reading of maps and drawings which, whilst necessary, requires the complementary understanding of place through experiential perception. The perception of place, however, is largely based on the explicitly perceivable elements defining space, hence many opportunities for innovative use of space and transformation of space into place are implicitly disregarded. The opportunities that exist particularly in Atopia (Menin Constructing Place: Mind \& Matter 2003) and Lost Spaces (Trancik Finding Lost Space 1986) are often missed. This paper posits that in order to develop environmentally sustainable architecture, the concept of adaptive reuse of existing space, in addition to materials and technology, is fundamentally necessary. For this to happen, however, architects need to develop qualities of perception that engage with the multiple, intangible, layers of space while at the same time being able to see opportunities for adaptive reuse of anti-space, "atopia" or lost space. The development of such perceptual abilities, however, requires a re-look at architectural pedagogy and methods of spatial analysis.

Keywords: environmental sustainability, eco-efficiency, urbanism, spatial waste, perceptual analysis, adaptive reuse, resilience, architectural pedagogy, interdisciplinarity.
\end{abstract}




\section{Introduction}

Design education in architecture and urbanism is becoming increasingly concerned with sustainability. Over the past two decades curricula and design projects have been significantly shaped by concerns with environmental sustainability, in particular. The 1992 Earth Summit in Rio de Janeiro highlighted, in broad terms, a very critical reality, that the vitality of our existence cannot be sustained if man and his endeavours continue to dominate and "tame" nature. The repercussions of the summit filtered across the globe and it was primarily the reason for the concept of "eco-efficiency" being touted as a new strategy for responding to the environmental damage caused by the modes of production and man's domination of nature (McDonough and Braungart [4]).

McDonough and Braungart [4]) refer to eco-efficiency as a concept that enables doing more with less. The efficacy of production became the primary focus in an attempt to lessen damage to the environment, caused by the rapid depletion of natural resources in the processes of industrial production. Besides the measurement of eco-efficiency, there is a determined effort to mitigate environmental damage by means of the reduction of waste. The current general icon for environmental sustainability, the triple $\mathrm{R}$ (reduce, recycle and reuse) has become widely recognised by society and the general level of environmental concern through mechanisms of recycling and reuse have increased exponentially in the past two decades. All this happens in a rational effort to reduce the impact of production on resource depletion and the production of waste. The reality of this situation, however, is that the mechanisms used to mitigate environmental damage do not solve the problems of resource depletion and the production of waste, but merely reduce the rates of such. Any process of production inevitably depletes some resources and produces waste. While certain industries successfully rehabilitate nature and transform industrial waste into useful products, other industries such as construction produce significant amounts of waste that rarely gets reused. Similarly, the creation of urban spaces and systems results in natural resource depletion as well as waste. While the production of industrial products, including built structures result in obvious and tangible waste, the creation of urban spaces results in spatial waste, in addition to product wastage.

While "sustainability tools" have been developed for industrial production and construction, the measurement of spatial waste has not been a significant focus of eco-efficiency measurement. Similarly, while recycling, reduction and reuse have been implemented in the production and use of industrial products, the spatial waste resultant from urban development has not featured adequately on the ecosustainability agenda.

This paper looks into ways in which spatial wastage caused by urban development may be vitalised by incorporating the concept of adaptive reuse. The principle problem, though, is difficult as the task requires the ability to perceive what is immaterial and unintended. It is argued that, while the analytical tools for measuring the eco-efficiency of buildings are quite developed and readily accessible as software applications, the "measurement" of spatial waste can only be effected through perceptual processes. In addressing this problem of spatial 
waste production, this paper posits that there needs to be a pedagogic shift in both academic curricula and professional practice in order to develop complex perceptual skills for the adaptive reuse of urban spatial waste.

\section{Defining urban spatial waste}

While waste from industrial production is an inevitable product of the process of production, urban spatial waste emanates from a dialectical opposite process. Urban spatial waste occurs as a direct result of the imposition of development on the natural landscape or historic urban fabric of existential spaces. Development, in this sense, does not merely refer to the physical construction of buildings and spaces but also socio-economic and political constructs and dynamics of place. What may have been a vibrant social community may have become a derelict ghetto, due to changes in social and economic realities or political structures.

The concept of spatial waste includes both open space, built space and the spaces defined by built form. Trancik [7]) refers to the term "lost space" in order to define what is referred to as spatial waste in this paper. The "residual" spaces that are left over from the act of design take various forms such as undefined and inappropriately scaled space between buildings and structures, the spaces left over beside motorways and railway lines, large tracts of land left for parking lots, and storage, among others. While degradation of buildings may be largely due to changes in social, economic and political structures, the creation of urban spatial waste is primarily due to the imposition of structure on the landscape, which includes the existing urban fabric. This artificial imposition on the existing fabric, which results in the creation of spatial waste, is ironically the wilful act of professionals that are trained in architecture and urban planning. Much of this residual space is created at the time of design and construction, while other lost spaces are created due to changes in social, economic and political dynamics. Albert Park in Durban, South Africa is one such space.

Albert Park (indicated in dotted circle at the top of Figure 1), established during the heydays of the apartheid era, was once a lively place for the privileged "white" people of Durban to meet and play. Political change however has changed the dynamics around the park and as such, the park has redefined itself as a wasted space that is often associated with illegal trade and activity. Referring back to Figure 1, railway tracks through Berea Station, has created a "dead edge" of lost space along the Western edge of Albert Park while a railway track and motorways between the waterfront and the park, has created another strip of lost space. The map further reveals a system of motorways that define certain edges and cut through certain districts, as in the Warwick Junction Precinct (indicated in dotted circle at the bottom of Figure 1) which has existed since the early development of the city. From reading the map (Figure 1), it may be assumed that there is a high degree of residual spatial waste due to the imposition of transport systems on the urban fabric. This is certainly true when evaluating the area using the traditional methods of urban analysis, such as figure ground drawings and maps. Perhaps the reality of "place" may also support the assumption, based on the abstract study of maps and drawings. This paper, however, posits that the analysis of spatial waste 
has to be factored into eco-efficient urbanism and architecture, and that a level of in-depth perception of space has to complement the established tools of spatial analysis and eco-efficiency that built environment professions utilise.

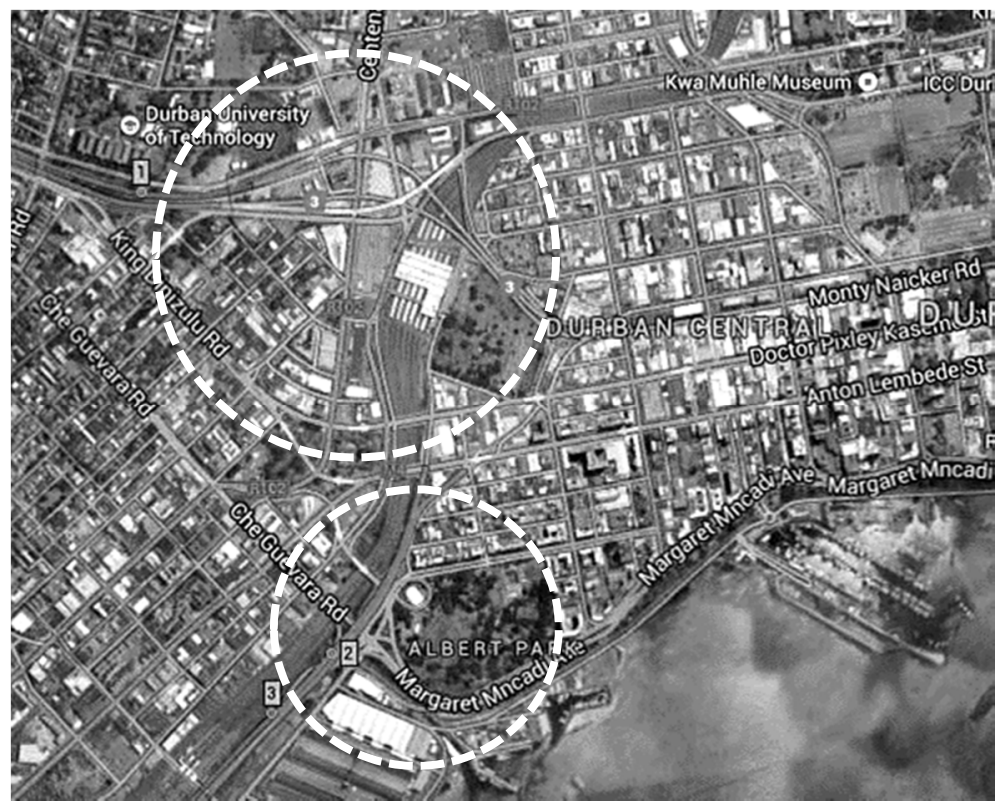

Figure 1: Aerial map indicating Albert Park and Warwick Junction Precinct (adapted from Rosenberg [6]).

\section{Dominant methods of urban spatial analysis}

Existing methods of spatial analysis tend heavily toward abstract convenience. Trancik [7]) outlined three approaches to urban analysis, namely, Figure Ground Theory, Linkage Theory and Place Theory, which he referred to as potential strategies for integrated urban design. Figure Ground Theory and Linkage Theory are primarily based on abstractions of physical contexts in the form of maps and drawings. Such theories are also a very convenient and quick method of analysing urban contexts in order to determine the form and structure of towns and cities, both historic and modern.

Nolli's map of Rome (Figure 2), in the form of a figure ground drawing, has been widely referred to in understanding the structure and form of ancient Rome. Figure ground drawings such as Nolli's map clearly show the structural order and the organic form of Rome. Furthermore, linkages between various ordering elements such as the Basilicas and Piazzas may be easily defined and determined. The detail of urban place, in its third dimension, however, cannot be determined by figure ground analysis. The reality of the city of Rome at human scale in place is what expresses the real quality of urban space. It is at this scale of place, 


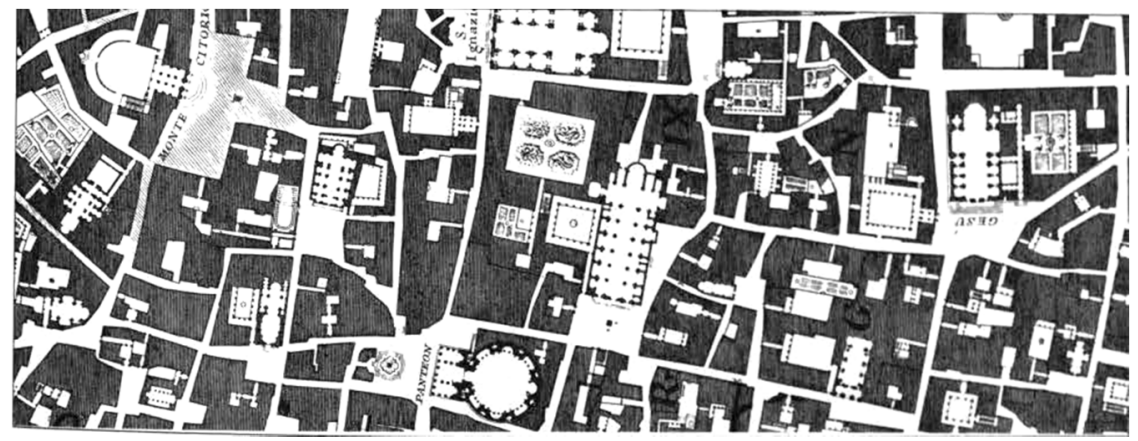

Figure 2: Nolli's Map of Rome 1748 (Trancik [7]).

which is defined by the material and proportional expression of architecture that connects people to urban space, which consequently defines place.

Lynch [3]) posits a Place Theory which is based on the psychological mapping of urban space. Lynch defined a set of elements that characterise urban place, namely, paths; edges; districts; nodes and landmarks. Orientation and way-finding which was structure by the coherent order of structuring elements, was the focus of Lynch's theory. The visual quality of space is what defined its legibility and the perceivable form of the city, according to Lynch.

The three theories of urban analysis discussed are convenient for the structural analysis of urban spaces. Even Lynch's place theory relies on structural abstraction in order to define and interpret urban place. These theories, however, somehow disregard the vitality of urban space and place, which is determined by the interaction of human beings with and within the urban environment. The definition of structural/elemental /material qualities of space as outlined by the above theories are referred to, in this paper, as the tangible qualities or the tangible layers of context. These layers are all determined and defined by structural order and analysed by using methods referring to the identification of structural order. This paper argues that there needs to be a qualitative method of analysis of urban context which transcends the "artificial" structural ordering approach, and looks further into the intangible layers of context.

\section{An alternate pedagogic approach towards sustainable urban spatial analysis and development}

The preceding section briefly outlined three structural approaches to urban space analysis and urban design. The methods are an effective and convenient way for abstract analysis of context and the design of "artificial" cities; cities defined by design and order. Alexander [1]) referred to the artificial city as a "tree" structure, and alternatively, the natural city as a "semi-lattice". The tree structure of the artificial city comprises various independent systems that have limited capacity for growth and very little, if no overlaps. The controlled and structured approach to city design, as discussed in the previous section, adopts literal legibility and 
determinism rather than complexity and indeterminism. The semi-lattice structure of the natural city, on the other hand is based on interdependent systems that work in a kind of eco-systemic synergy within a greater holistic, indeterminate and complex mega-system, namely, the city. The semi-lattice metaphor further reveals a multi-layered complexity of the urban context, which comprises both tangible/structural layers as well as the intangible layers.

The complexity of the city as a system of interconnected micro-systems is elaborated upon by Dovey [2] who refers to Assemblage. "An assemblage is a whole that is formed from the interconnectivity and flows between constituent parts - a socio-spatial cluster of interconnections between parts wherein the identities and functions of both parts and wholes emerge from the flows between them." (Dovey [2]). An assemblage therefore cannot merely be defined by literal, structural or material qualities of space. It is the inter-connective relativities between the physical elements that define space and the "soft" socio-spatial systems that co-exist in order to define an assemblage, which must therefore be dynamic, spontaneous and indeterminate. The rhizomic informalities that may not be clearly visible in the visual or aesthetic surface dimension, determine the complex vitality of urban space. Urban complexity is therefore the composite system of tangible layers and the intangible layers that define space.

This leads the discussion to spatial analysis of the intangible /immaterial/ and "in-between" urban spaces that cannot be "seen" when utilising the traditional methods of urban analysis which are based on abstract methods. The reality of urban life in cities is that the inherent multicultural socio-economic dynamics of urban dwellers is what determines the vitality of urban place. It is this opportunistic act of dwelling within urban spaces that often results in urban vitality that could never have been designed in any abstract way. Need and resilience to socio-economic and political forces ultimately ignites creativity in the identification of space and the utilisation thereof, and this often happens rather informally. It is those spaces that are missed by the traditionally trained "design eye" which somehow develop into vibrant urban places and in cases, such as the Warwick Junction Precinct in Durban, as parallel cities or dual CBDs.

\section{Urban resilience: the case of Warwick Junction Precinct (WJP)}

While political change in South Africa had a negative effect at Albert Park, the Warwick Junction Precinct (WJP) developed rather paradoxically. British and Boer administrations during the colonial period, tried to control, confine and undermine non-white business which started to flourish in the city after the arrival of Indian labourers under the indenture system. Colonial administration actively developed increasingly segregationist and prejudicial laws that strictly controlled and restricted the settlement and trading opportunities of Indians and Africans in Durban. The Dealer's Licence Act of 1897 resulted in the formalisation of a dual racialised CBD with a corresponding dual transport system. Indians were not allowed to trade in the three main streets, through various policies, licensing and disenfranchisement laws. During the 1930s some of the Indian trade moved 
to Warwick Avenue and the establishment of a new Early Morning Market, also known as "Squatters Market", in close proximity to the station. Indian business started to thrive and Indian residential property ownership increased around this area. In 1973, under the Group Areas Act this precinct became proclaimed as an Indian Group Area, which allowed Indians to trade in this part of the city but not to live in the city. As a consequence, about 12000 Indians had to vacate the city. However, by 1981, only half had moved and by 1983 Indians were, once again, allowed to live in the Grey Street precinct (Rosenberg et al. [6]).

The establishment of an Indian Market and the Berea Station eventually resulted in the development of Warwick Junction as a dual CBD, referred to by some, as the "Black or Indian CBD".

The Indian CBD contrasted in character to its White counterpart which was characterised by tall buildings, typical of many American cities. This was as a result of less financial resources by the predominantly Indian family owned businesses. Furthermore the WJP developed a character defined by a rich mix of uses (as indicated in Figure 3), from commercial to educational to residential to religious and various other functions. This precinct was all about sustaining the culture of society - it was a human/people-centred urban environment that developed responsively and resiliently, over time. In this context, the elements of urban design are complemented by the play and evolution of urban culture.

The typology of the Grey Street buildings were low rise with shops at street level and residential units above (Figure 4), still evident today.

Formality and informality exist in perfect harmony (Figure 5) and are complementary rather than competitive. Indeed, many of the families of the original shop owners continue to trade, mostly under the same trade name, despite changes in political structures and city governance.

This precinct is characterised by the informal "colonisation" of any available undefined space such as wide pavements and alleys between buildings (Figure 6).

The post-apartheid era has seen a flourish in black informal business and the Warwick Junction Precinct has established itself as a major economic (mainly informal) centre that enjoys both the interaction with daily commuters and the large groups of tourists who are enchanted by this special alternate CBD on a daily basis.

The precinct is also characterised by the adaptive reuse of residual space, abandoned bridges (Figure 7) and almost every "un-designed" space has been colonised by traders.

What is defined as lost space and atopia, which are largely the by-products of design, become activated by human dwelling. These spaces become adaptively reused in ways never imagined (Figure 8). This, in turn, redefines eco-inefficient spaces, that were wasted spaces, into eco-efficient spaces that become receptacles to the interconnected, dynamic play of urban life. The result is a consequential urban densification in new and unexpected ways; a somewhat paradoxical urban definition as what was intended to restrict opportunity for marginalised communities, transformed into the most vibrant precinct of the city of Durban. 


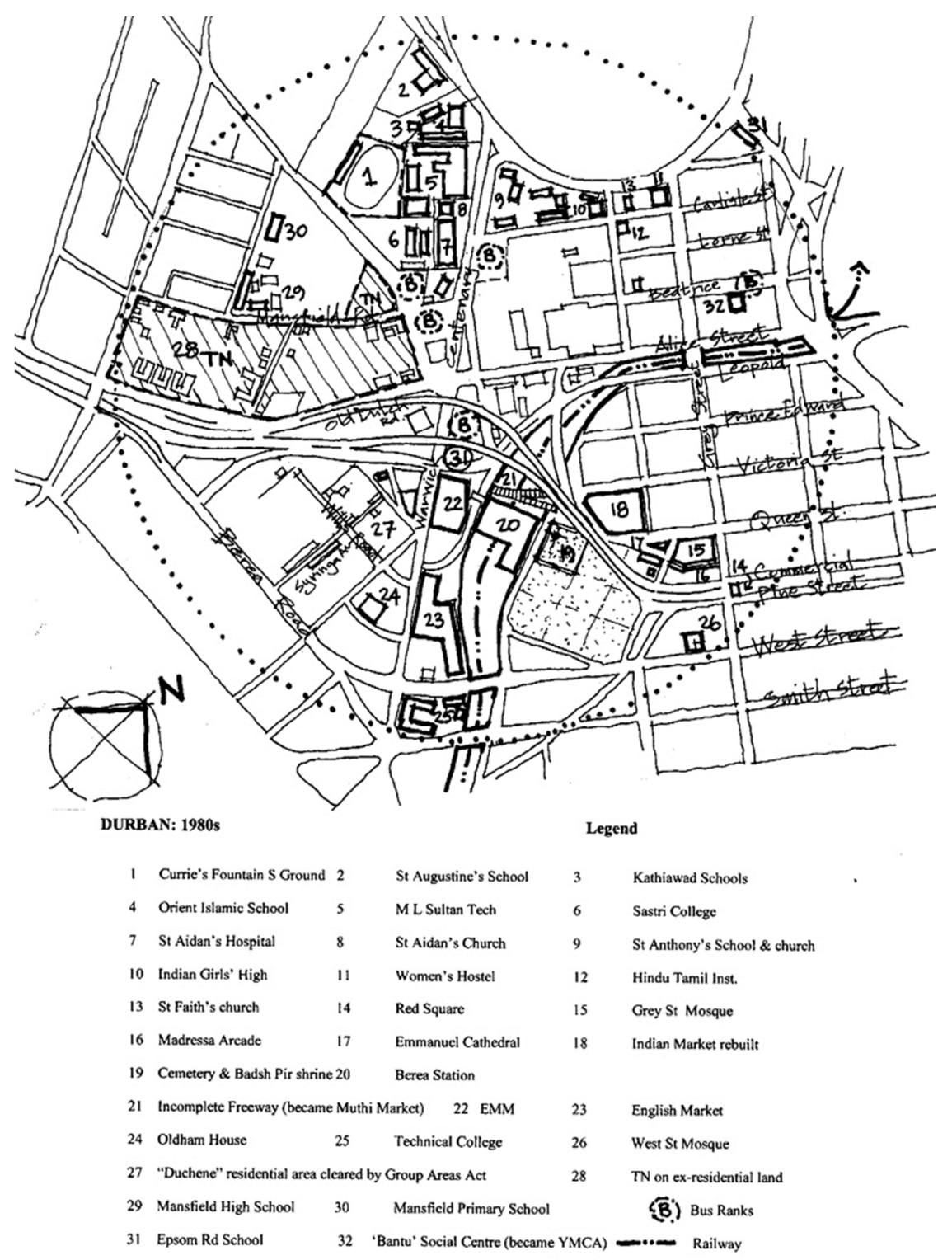

Figure 3: Warwick Junction during the Colonial Period, 1970s-1980s (adapted from Rosenberg et al. [6]). 


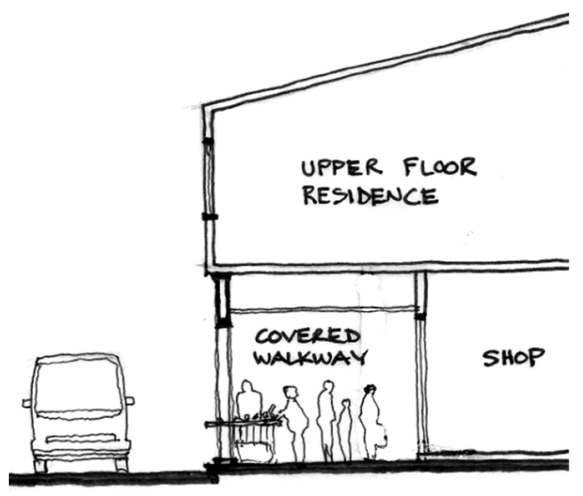

Figure 4: Section through typical shop/residence unit in the Grey Street Precinct (author 2014).

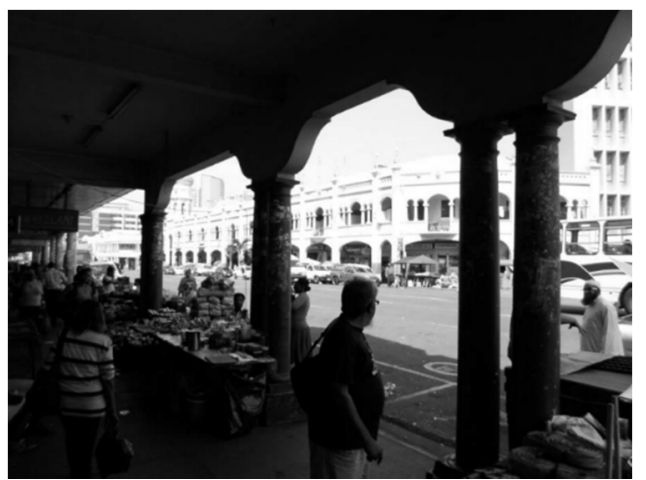

Figure 5: Informal trade in covered pavement walkways outside formal shops in the Grey Street/Warwick Junction Precinct (author 2013). 


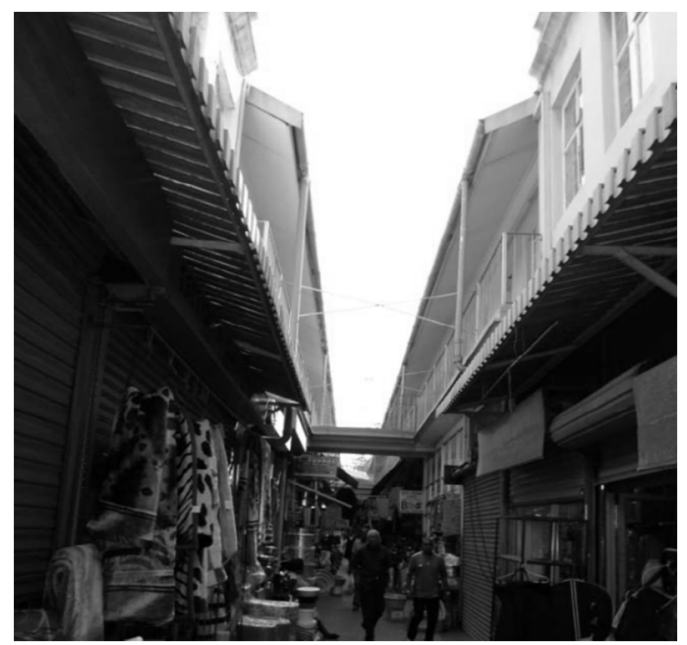

Figure 6: Informal trade on alleys between buildings outside formal shops in the Grey Street/Warwick Junction Precinct (author 2013).

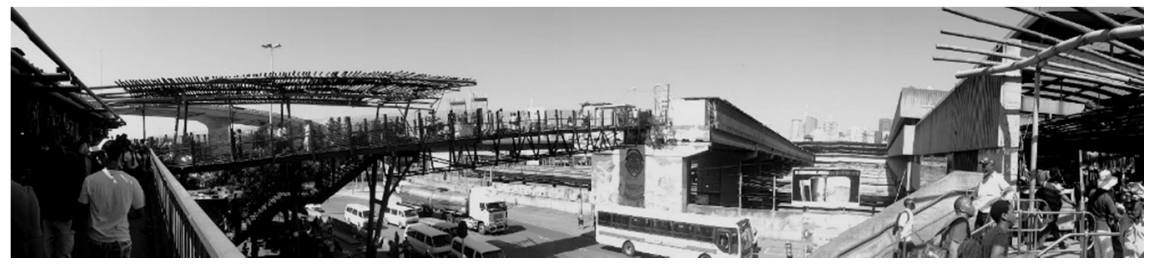

Figure 7: Informal trade along link bridges, Warwick Junction Precinct (Govender, V., Maharaj, S., Pillay, D., Ramchund, A.: DUT, Third Year Design Students 2014).

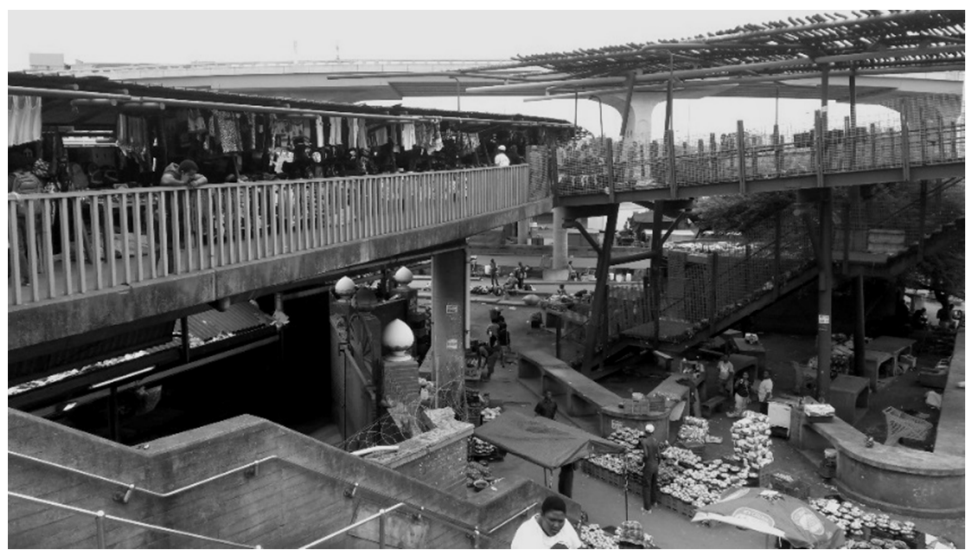

Figure 8: Informal trade in residual spaces around the Berea Station, Warwick Junction Precinct (Del Monaco 2014). 


\section{An interdisciplinary pedagogic approach to urban analysis and design}

The above analysis has revealed that the traditional methods of spatial analysis fall short of a level of perception, that of the potential urban vitalisation as a consequence of adaptive dwelling. There is an adept ability of designers to manipulate the physical, and material qualities of space and the elements that define such space, however, there is a general lack of focus on human social factors that activate urban spaces. There is a disconnection between ideology, practice and society/environment. This paper looks toward a humanistic approach to the design of urban spaces in order to create holistic urban environments that are characterised by the layering of the tangible and the intangible attributes of space, with people as the central focus. The design of urban spaces invariably involves the creation of artificial space. However, it is argued that a collaborative and interdisciplinary approach to design is necessary for the creation of humanistic urban spaces that are economically, socially and environmentally sustainable. For this to happen, designers have to step out of their "silos" and engage in an interdisciplinary way, with the urban problem and the dynamics of place as the focus of any design process.

An interdisciplinary approach to design involves the integration of multiple domains which include, multiple intelligences, multiple cultures and multiple perspectives. Any urban design would invariably emanate from negotiated solutions that are never determinate but rather, responsive, adaptive and, therefore, indeterminate. As such urban place cannot be the result of any singular act of will or genius, but rather an evolutionary process of the development of interconnected systems that develop organically over what has to be a "loosely" designed urban context. This requires that designers give up their expert cultures and start to engage with urban problems in a collaborative way as the perception of the potential for urban life cannot be achieved in disciplinary silos, by professionals who have been traditionally trained. The people that dwell in urbanism have to be part of this interdisciplinary engagement. People, Place and Time become the vital constituents of sustainable urban design. Time is a concept that has to be interpreted as dynamic and not static or frozen in any particular moment. Social/cultural and economic structures evolve over time and this evolution cannot be predicted beyond the short term. Urban design and architecture therefore has to factor in the evolutionary process of time and this is the real and exciting challenge for academics and practitioners today.

\section{Conclusion}

The paper looked at the value of spatial perception in the definition of urban spatial analysis and design, as a complementary method to the analytical method of measuring eco-efficiency of buildings and products. It became apparent that traditional training in urban design and architecture does not sufficiently develop such perceptual skill, mainly due to the "silo" approach to education and, hence, practice. The design and construction of urban environments, therefore, inevitably 
result in spatial waste. The resilience and need of urban dwellers somehow find ways of adaptively reusing such spaces and residual/wasted space becomes vitalised through socio-spatial engagement that is often determined by the need to sustain the livelihood of people in urban spaces. This adaptive reuse of space is a vital form of eco-efficiency as well as social and economic sustainability. The pedagogic challenge, thereby, presented requires a shift in the pedagogic approaches to architecture and urbanism, which require a collaborative and interdisciplinary mode of engagement with urban design problems. The ultimate outcome would be to develop vibrant and sustainable urban spaces that respond to people, place and time.

\section{References}

[1] Alexander, C., A city is not a tree. Design, London Council of Industrial Design, 206, pp. 1-17, 1966.

[2] Dovey, K., Informal urbanism as resilient assemblages; Melbourne School of Design, University of Melbourne, Online. www.indahwidiastuti911. files.wordpress.com. Accessed: 26 February 2014.

[3] Lynch, K., The Image of the City, The Technology Press \& Harvard University Press: Cambridge, pp. 1-117, 1960.

[4] McDonough, W. \& Braungart, M., The next industrial revolution. The Atlantic, October 1998.

[5] Menin, S., Constructing Place: Mind \& Matter, Routledge: London and New York, 2003.

[6] Rosenberg, L., Vahed, G., Hassim, A., Moodley, S. \& Singh, K., The Making of Place: The Warwick Junction Precinct, Durban University of Technology Press: Durban, 2013.

[7] Trancik, R., Finding Lost Space, Van Nostrand: New York, pp. 97-124, 1986. 